\title{
Giant Coronary Artery Aneurysms in Kawasaki Disease Detected by Multi Detector Computed Tomographic
}

\author{
Oktavia Lilyasari, Poppy S Roebiono, Radityo Prakoso, Anna Ulfah Rahajoe, \\ Indriwanto Sakidjan, Ganesja M Harimurti
}

\begin{abstract}
Kawasaki disease is an acute, self -limited vasculitis of unknown etiology that occurs predominantly in infants and young children. The major sequele of Kawasaki disease are related to the coronary arterial system. Cardiac imaging is a critical part in evaluation of all patients with suspected Kawasaki disease. Multi Detector CT (MDCT) provides a safe non-invasive approach to accurately delineate coronary artery anatomic structure. We report a case of a 6 years old boy with history of Kawasaki disease. Multi detector CT scan showed giant aneurysm at proximal LAD, proximal RCA and medium aneurysm at proximal LCX. Patient then treated with the anticoagulant therapy.
\end{abstract}

(J Kardiol Indones. 2013;34:133-5)

Keywords: Kawasaki Disease-Multi Detector CT scan

\section{Case Report}

1 6 years old boy was diagnosed with Kawasaki disease 3 months before admission and previously treated with intravenous immunoglobulin. The patient was sent to us with suspicion of developing coronary aneurysm. The echocardiography examination found dilatation of Left Main (LM) $2.7 \mathrm{~mm}$ and coronary aneurysm involving

\footnotetext{
Alamat korespondensi:

Dr. Oktavia Lilyasari, Departemen Kardiologi dan Kedokteran Vaskular FKUI, Pusat Jantung Nasional Harapan Kita. Jakarta. E-mail: oktavia_lilyasari@yahoo.com
}

Left Anterior Descending (LAD) and Left Circumflex (LCX) measuring $4 \mathrm{~mm}$ in diameter and Right Coronary Artery (RCA) measuring $4.5 \mathrm{~mm}$ in diameter.

For further evaluation we performed MDCT on this patient. The patient was asymptomatic at the time of the CT examination. Coronary CT angiography is performed as an electrocardiographically gated contrast medium-enhanced CT scan of the heart. It showed normal diameter of LM measuring $2 \mathrm{~mm}$ in diameter. The coronary aneurysms involving the proximal of LAD measuring 8.2 in diameter and $20.6 \mathrm{~mm}$ in length, proximal LCX measuring $6.4 \mathrm{~mm}$ in diameter and $18.7 \mathrm{~mm}$ in length, and proximal RCA measuring $10.9 \mathrm{~mm}$ in diameter and $30.8 \mathrm{~mm}$ in length (Figure 1). Distal part and branches of these coronary arteries 
are normal in diameter (Figure 2). The Patient is currently stable without any clinical symptom and treats with the anticoagulant therapy.
Coronary artery aneurysms and ectasia are characterized by an abnormal dilatation of a coronary artery and aneurysm is a focal dilatation of the vessel. ${ }^{3}$ In the

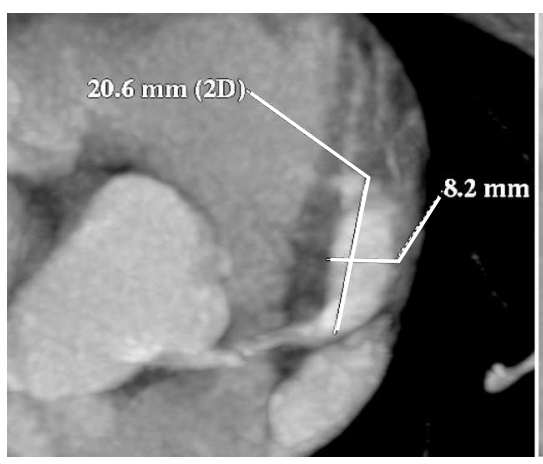

(a)

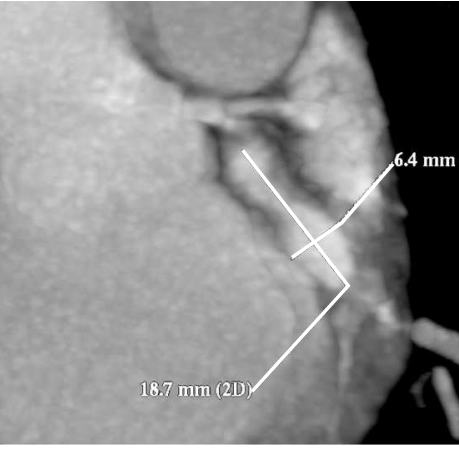

(b)

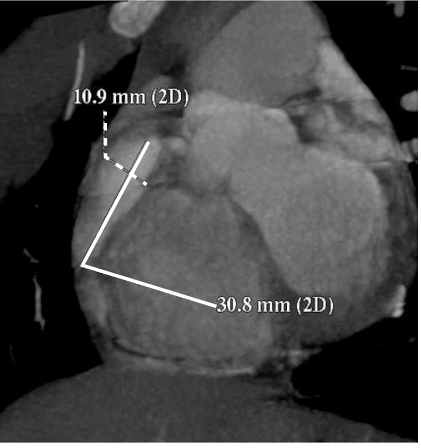

(c)

Figure 1. Contrast enhanced CT demonstrating : (a). A giant aneurysm at the proximal LAD. (b). A medium aneurysm at the proximal of LCX. (c). A giant aneurysm at the proximal of RCA.
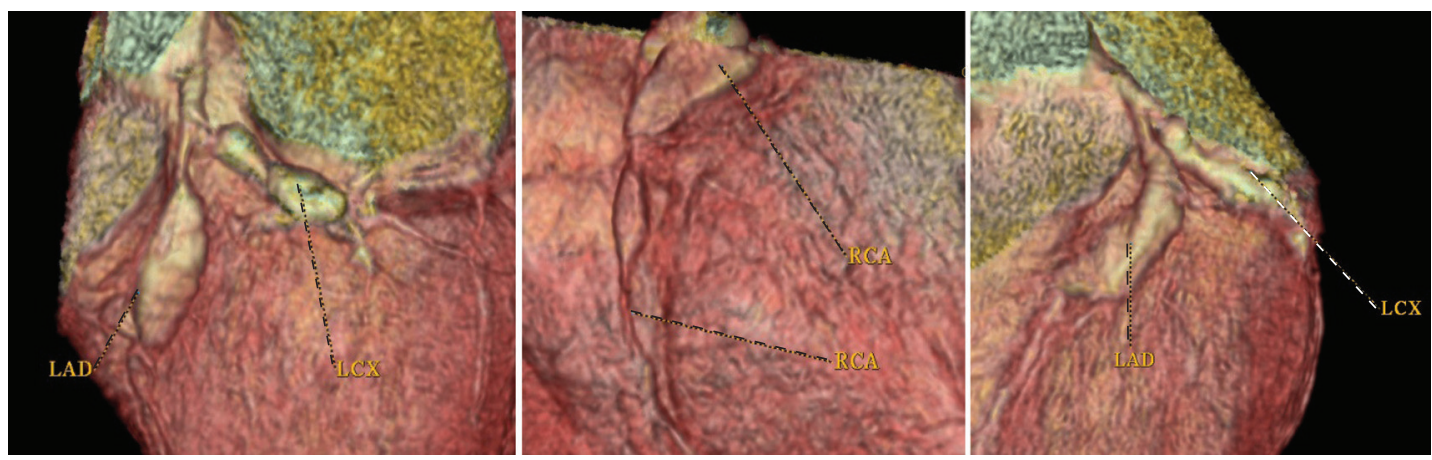

Figure 2. Volume Rendering three-dimensional (3D) coronary arteries show the location and morphology of coronary aneurysm.

\section{Discussion}

Kawasaki disease is an acute, self -limited vasculitis of unknown etiology that occurs predominantly in infants and young children. First described in Japan in 1967 by Tomisaku Kawasaki. Kawasaki disease is characterized by fever, bilateral non-exudative conjunctivitis, erythema of the lips and oral mucosa, changes in the extremities, rash and cervical lymphadenopathy. Cardiovascular involvement is the most serious complication. If not treated well, the patients have substantially increased risk of developing coronary artery aneurysms. Acute inflammation begins in the intima and adventitia, and then involve the media. Disruption of the elastic lamina begins to weaken the arterial wall and consequently, coronary artery aneurysms develop in $15-25 \%$ of patients. ${ }^{1,2}$ last American Heart Association statement, aneurysms were classified as a small ( $<5 \mathrm{~mm}$ internal diameter), medium (5-8mm internal diameter), or giant $(>8 \mathrm{~mm})$ internal diameter. Giant are associated with a greater morbidity and mortality. Stenosis, thrombosis, or occlusion may develop at either end of the aneurysm. Myocardial infarction from stenosis or thrombosis in the area of an aneurysm is the principal cause of death from Kawasaki disease. ${ }^{1}$

The major sequalae of Kawasaki disease are related to the cardiovascular and more specifically the coronary arterial system. So cardiac imaging is a critical part in evaluation of all patients with suspected Kawasaki disease. Echocardiography is an ideal non invasive examination and has a high sensitivity and specificity for detection of abnormalities of the proximal Left Main Coronary Artery (LM) and 
Right Coronary Artery (RCA). It is important to recognize the limitation of echocardiography in the evaluation and follow up of patients with Kawasaki Disease. The visualization of coronary arteries becomes progressively more difficult as a child grows and body size increase. Other modalities such as angiography, intravascular ultrasound (IVUS), trans-esophageal echocardiography, cardiac magnetic Resonance Angiography (MRA) and ultrafast computed tomography (CT) may be of valuable in the assessment of selected patients. 1,4

Nowadays, state of the art Multi Detector CT (MDCT) technology with higher spatial and temporal resolution provides a safe and valuable non-invasive approach to accurately delineate coronary artery anatomic structures. ${ }^{3,4,5}$ MSCT can depict all the morphologic alteration of Kawasaki coronary disease showing abnormal wall thickness and characterize plaque component. These features may allow a noninvasive monitoring with clinical impact in optimizing anti platelet or anti-inflammatory therapy. ${ }^{6,7}$

This case illustrates the coronary aneurysm associated with history of Kawasaki syndrome. It can be occurred in asymptomatic 6 years old patient few months after the acute phase even he had been treated with immunoglobulin therapy. Accurate visualization of coronary arteries on echocardiography became difficult in this patient since this child getting bigger. Hence, Multi Detector Computed Tomography coronary angiography was used to evaluate the coronary artery complications and it showed clearly the giant aneurysm of the coronary arteries. Regarding of this finding we should optimize the anticoagulant therapy and perform regular follow up to monitor this patient.

\section{Conclusion}

Reviewing of coronary artery in asymptomatic patient with history of Kawasaki disease is still needed. Multi Detector Computed Tomography coronary angiography represents an ideal non-invasive imaging modality for diagnosis and follow up of the coronary arterial complication of Kawasaki disease as adjunctive modality of echocardiography. Its use in the management of Kawasaki disease should be considered.

\section{References}

1. Newburger j.W., Takahashi M, Gerber M.A., Gewitz M.H., Tani L.Y., et al. Diagnosis, treatment, ad Long-term management of Kawasaki Disease : A Statement for Health professional from the Committee on Rheumatic Fever, Endocarditis and Kawasaki Disease, Council on Cardiovascular Disease in the Young, American Heart Association. Circulation 2004;110:27472771

2. Kim J.A., Chun E.J., Choi S.I., Kang J.W., Lee J, Lim T.H. Less Common Causes of Disease involving the coronary arteries : MDCT Findings. American Journal Radiology, 2011;197:125130

3. Zamudio m.D., Perez U.B., Zarza M.C.H., Gonzales A.M., Rosas E.A., Zambrana-Balta G.F.,Kimura-Hayama E.T. Coronary Artery Anneurysms and Ectasia : Role of Coronary CT Angiography. Radiographics.rsna.org, November-December 2009;1939-1954

4. Chao BT., Wang XM., Wu LB., Chen J., Cheng ZW., Wu DW., Duan YH. Diagnostic value of Dual Source CT in Kawasaki Disease. Chin Med J. 2010 Mar 20; 123(6):670-4

5. Xing Y., Wang H., Yu X., Chen R., Hou Y. Assessment of coronary artery lesion in children with Kawasaki disease : evaluation of MSCT in comparison with 2D echocardiography. Pediatr Radiol 2009 Nov;3(11):1209-15

6. Renzulli M, Piovaccari G, Fattori R. Potential of multislice CT in the follow up Kawasaki coronary disease. Eur Heart J 2006(20):2384

7. Manghat N.E., Morgan-Hughes G.J., Coc D.I., Roobottom C.A. Giant Coronary artery aneurysm secondary to Kawasaki disease: diagnosis in adult by multi detector row CT coronary angiography. The British Journal of Radiology 2006(79):e133136 